\title{
Ribosome crystallography: catalysis and evolution of peptide-bond formation, nascent chain elongation and its co-translational folding
}

\author{
A. Bashan and A. Yonath' \\ Department of Structural Biology, Weizmann Institute, 76100 Rehovot, Israel
}

\begin{abstract}
A ribosome is a ribozyme polymerizing amino acids, exploiting positional- and substrate-mediated chemical catalysis. We showed that peptide-bond formation is facilitated by the ribosomal architectural frame, provided by a sizable symmetry-related region in and around the peptidyl transferase centre, suggesting that the ribosomal active site was evolved by gene fusion. Mobility in tunnel components is exploited for elongation arrest as well as for trafficking nascent proteins into the folding space bordered by the bacterial chaperone, namely the trigger factor.
\end{abstract}

\section{Introduction}

A ribosome is a ribozyme polymerizing amino acids, capable of reliable translation of the genetic code. It is a complicated riboprotein assembly made of two subunits. The small subunit (30 S in prokaryotes) provides the decoding centre and controls translation fidelity. The large subunit $(50 \mathrm{~S}$ in prokaryotes) enables the three catalytic events required for the formation of nascent proteins: peptide-bond formation, amino acid polymerization and peptide release. It also contains the protein exit tunnel and the machinery supporting early co-translational nascent protein folding. The recent high-resolution structures of ribosomal particles [1-5] were exploited for providing structural bases for the various ribosomal functions. Nevertheless, the ribosomal catalytic events are still not fully understood.

The findings that (i) the 'fragment reaction' in which minimal substrates such as puromycin, a universal inhibitor mimicking the tip of the tRNA $3^{\prime}$-end, can be catalysed by naked ribosomal RNA [6,7], (ii) the localization of the PTC (peptidyl transferase centre) is in a protein-free environment, rich in conserved nucleotides [4,5,8-10], (iii) the usage of puromycin derivatives bound to the partially disordered large subunit from Haloarcula marismortui (H50S) [8] together with a compound presumed to resemble the reaction intermediate [11], and (iv) the assumption that ribosome catalysis resembles the reverse mode of serine proteases, led to a hypothesis according to which ribosomal nucleotides participate in the chemical events of peptide-bond formation [8]. Various biochemical and mutational results [12-14], such as the finding that PTC in crystalline H50S does not resemble active conformation [15] and that in some complexes, A2451

Key words: nascent chain elongation, peptide-bond formation, positional catalysis, ribosome, substrate-mediated catalysis, trigger factor.

Abbreviations used: PTC, peptidyl transferase centre; TF, trigger factor

${ }^{1}$ To whom correspondence should be addressed (email ada.yonath@weizmann.ac.il).
(Escherichia coli nomenclature throughout), proposed to be the main player in the reverse-serine-proteases reaction, points away from the reaction centre [11], and the involvement of nucleotide A2602 in tasks other than peptide-bond formation, such as nascent peptide release [16] and anchoring tRNA A-site to P-site passage [9,10], challenged this hypothesis and provided no grounds for expecting that the complex assembly called ribosome catalyses protein biosynthesis by the reverse of a common enzymatic mechanism.

A symmetry-related region, containing 180 nucleotides (Figure 1a), was identified in all known structures of the large subunit, within the otherwise asymmetric ribosome [9]. The linkage between the symmetry axis and A-site tRNA positioning, as observed in the whole ribosome from Thermus thermophilus, T70S [4] and in the large subunit from Deinococcus radiodurans, D50S [9], revealed that the ribosome provides a striking architectural frame, ideal for amino acid polymerization $[9,10]$. Further experiments showed that mutagenesis of four universally conserved nucleotides of the PTC innermost layer, A2451, U2506, U2585 and A2602 (Figure $1 \mathrm{~b}$ ), does not affect the rate of peptide-bond formation by intact aminoacyl tRNAs, but substantially changes the rate of peptide release, consistent with the notion of PTC involvement in two distinct chemical reactions: aminolysis (peptidebond formation) and hydrolysis (peptide release) [17]. Later experiments revealed that the chemical component of peptide-bond catalysis is mediated by the substrate, namely the P-site tRNA, of which the $2^{\prime}-\mathrm{OH}$ of $\mathrm{C} 76$ plays an essential role [18].

Positioning of reactants in an orientation suitable for chemical reactions is performed by almost all biocatalysts [19], including the ribosome. Unlike enzymes catalysing a single chemical reaction, such as proteases, and similar to other polymerases, the ribosome provides the means for substrate motions required for the processivity of peptide-bond formation, namely for amino acid polymerization [20,21]. In the present study, we show that the ribosomal architectural 
Figure 1 | The rotatory motion

(a) A view down the symmetry region. Overlap of the RNA backbones of the symmetry-related region in T70S (Protein Data Bank accession no. 1GIY), D50S (PDB accession no. 1NKW) and H50S (PDB accession no. 1JJ2). The A-site subregion is shown in blue and the P-site in green. ASM and the derived P-site [9] are shown in blue and green, respectively. A detailed view of the symmetrical region inner part [colour code is as in (a)] with the position of the symmetry axis is shown (in red) with nucleotides within $15 \AA$ from the symmetry axis and the A- and P-site tRNA tips. The two RNA nucleotides (A2602 and U2585) anchoring the A- to P-site passage are highlighted. Note that these are also shown in (c). (b) A view perpendicular to the symmetry region, showing the ribosomal-RNA backbone (in grey) and the A- and P-site tRNA's 3'-ends, as in (a). The recently mutated four innermost nucleotides $[9,10,16,17]$ are coloured. (c) The space allocated to the rotary motion within the very crowded large subunit (RNA shown in grey). The rotatory motion is illustrated by snapshots of successive rotations of ASM (light blue) around the bond connecting the $3^{\prime}$-end with the rest of the tRNA molecule. The blue arrow indicates the direction of the rotatory motion from A- to P-site.


frame governs the positional as well as the chemical contributions to the catalysis of peptide-bond formation; describe the parameters dictating correct substrate placement; discuss evolution aspects implicated by the ribosomal function and point at a possible correlation between peptidebond formation, nascent protein progression, co-translational folding and cellular regulation.

\section{Positional catalysis contributions}

The PTC is an arched void with dimensions suitable for accommodating the $3^{\prime}$-ends of the A-and the P-site tRNAs (Figure 1c). It is located adjacent to the protein exit tunnel, within the sizable symmetry-related region that connects all ribosomal functional centres involved in amino acid polymerization: the tRNA entrance and exit regions, the PTC and its bridge with the decoding site. Each of the symmetryrelated subregion contains half of the PTC, namely either the $\mathrm{A}$ - or the $\mathrm{P}$-site, and the axis relating them by approx. $180^{\circ}$ rotation is located in the middle of the PTC (Figure 1a). In a complex of D50S with ASM, a $35 \mathrm{nt}$ oligomer mimicking the aminoacylated-tRNA acceptor stem, the bond connecting tRNA 3 '-end with the rest of the tRNA molecule overlaps the rotation axis [9]. This suggests that tRNA $A \rightarrow P$ site passage is a combination of two synchronized motions: a sideways shift of most of the tRNA molecules and a rotatory motion of the tRNA $3^{\prime}$-end within the PTC. 
Figure 2 | Peptide bond formation

(a) The D50S PTC cavity viewed from the subunit interface, together with ASM and its derived P-site tRNA [9]. The nucleotides known to form base-pairs with the A-site and P-site tRNA molecules are numbered. (b) The position of the tips of the A- and the P-site tRNAs, as obtained by applying the rotatory motion to ASM. Highlighted are the participants in peptide-bond formation: light blue, the amino group; red, the carbonyl oxygen; and foggy red, $\mathrm{2}^{\prime}$-OH of the P-site tRNA.
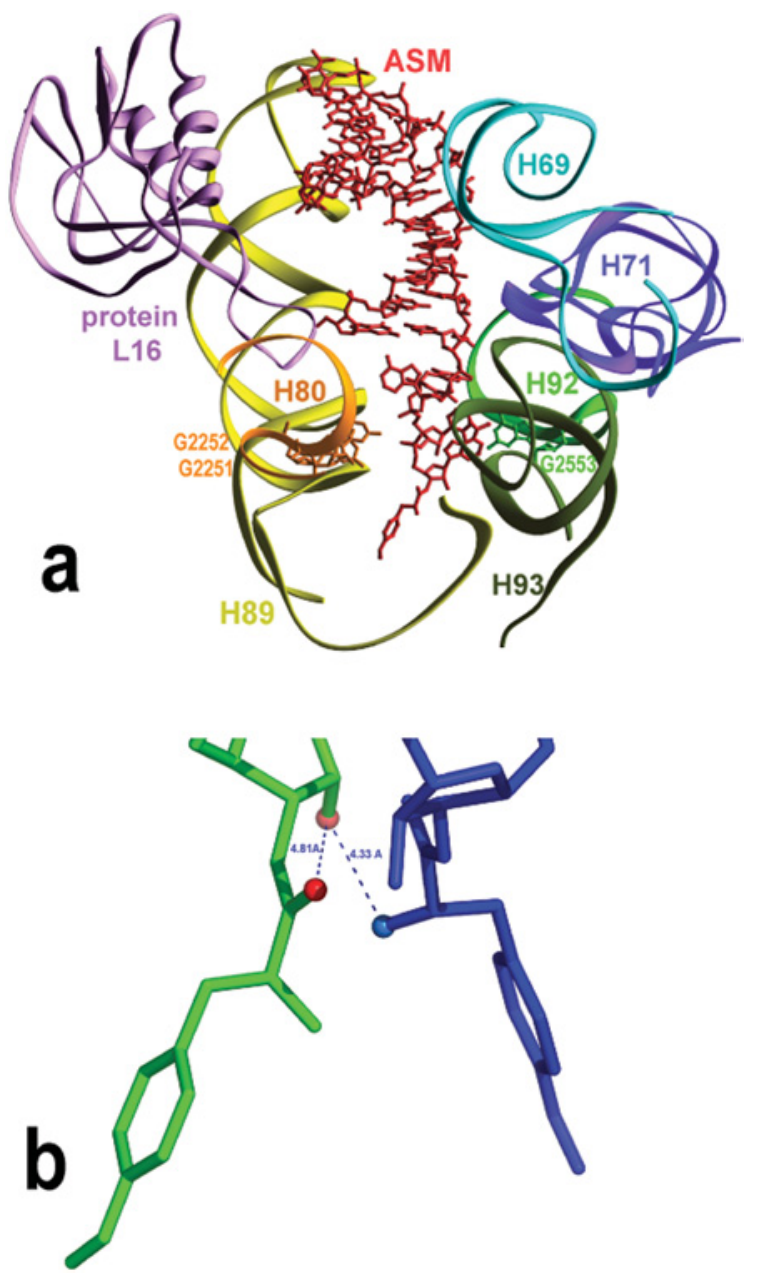

Simulation of this rotatory motion revealed that it is navigated and guided by PTC components and that its outcome is a stereochemistry appropriate for peptide-bond formation $[9,10]$ (Figure 1a). Furthermore, the location and the orientation of the peptide bond thus created is adequate for the subsequent ribosomal tasks, releasing the peptidyl tRNA and directing the nascent protein into the exit tunnel in an extended, $\beta$-like conformation, complying with the tunnel diameter at its entrance.

A remarkable PTC-conserved feature, comprising two successive guanines (G2251 and G2252), is capable of basepairing with the two tRNA 3 '-end universal cytosines (Figure $2 \mathrm{a}$ ). The rotatory motion guides the P-site tRNA to an orientation appropriate for the creation of these two base pairs. These, in turn, were found to be essential for the P-site
tRNA catalytic role in peptide-bond formation (Figure $2 \mathrm{~b}$ ) [18]. In addition, for initiating the rotatory motion, the initial P-site tRNA should acquire its functional orientation. Having two guanines at the P-site, compared with one at the A-site, seem to fulfil this requirement [21,22]. It appears therefore that the ribosomal architectural-frame governs the catalytic positional requirements and provides the means for the chemical catalysis. Hence, the two seemingly independent contributions to peptide-bond formation, namely the positional and the chemical catalysis, are integrated and the ribosome is responsible for substrate orientation, substratemediated chemical catalysis and for the processivity of the polymerization.

\section{Hierarchy of substrate placement and ribosome evolution}

Correlation was found between the rates of peptide-bond formation and the substrate type. Thus, in contrast to the unaffected rates when using tRNA substrates, significantly decreased rates were observed when using the minimal substrate puromycin [17], suggesting that the latter have to undergo additional repositioning. This decreased reaction rate is consistent with crystallographic results, showing diverse binding modes for minimal substrate analogues, all requiring rearrangements to participate in peptide-bond formation $[11,23]$.

Importantly, the universal C75-G2553 base-pair [17] is formed by all-bound substrate analogues. However, smoother rotatory motion can be performed by substrates placed correctly, namely by remote interactions with the upper part of PTC cavity [9,10]. Remote interactions cannot be formed by substrate analogues that are too short to reach the upper part of PTC cavity, as 'fragment reaction' participants [8,9], or when Helix H69, the remote-interaction mate at the PTC upper end, is disordered, as in the H50S structure $[1,8]$. It appears, therefore, that the CCA basepairing contributes to the overall positioning of the $3^{\prime}$-end of the aminoacylated tRNA, whereas the efficiency of peptidebond formation depends on the remote interactions.

The ribosomal symmetrical region hints that its active site is evolved by gene fusion of two separate domains of similar structures, each hosting a part of the catalytic activity. Consistently, substrate-mediated catalysis is rather rare in modern enzymology $[24,25]$. The lack of sequence identity between the two symmetrical subregions demonstrates a rigorous requirement of accurate substrate position that dictates the preservation of the three-dimensional structure regardless of the sequence. Importantly, the ribosomal protein L16, the only protein contributing to tRNA positioning [9], displays a conserved tertiary structure along with a diverged primary sequence, as observed first for the globin family [26].

Conservation of PTC nucleotides was correlated with the CCA universality. As described above, C74 and C75 universality of the cytosines in the tRNA $3^{\prime}$-termini (C74 and C75) is linked to their base-pairing mates in the PTC. Consistently, 
Figure 3 Dynamics in the tunnel opening

The tunnel opening (grey-violet-brown cloud) in D50S. The structures of L23 in H50S and D50S (HL23 and DL23 respectively) are superposed. Protein L39e that exists only in $\mathrm{H} 50 \mathrm{~S}$ (HL39e) in proximity to $\mathrm{H} 23$ is also shown. The arrows show the direction of the allosteric motion induced by TF binding to L23 globular side, at the ribosome exterior.

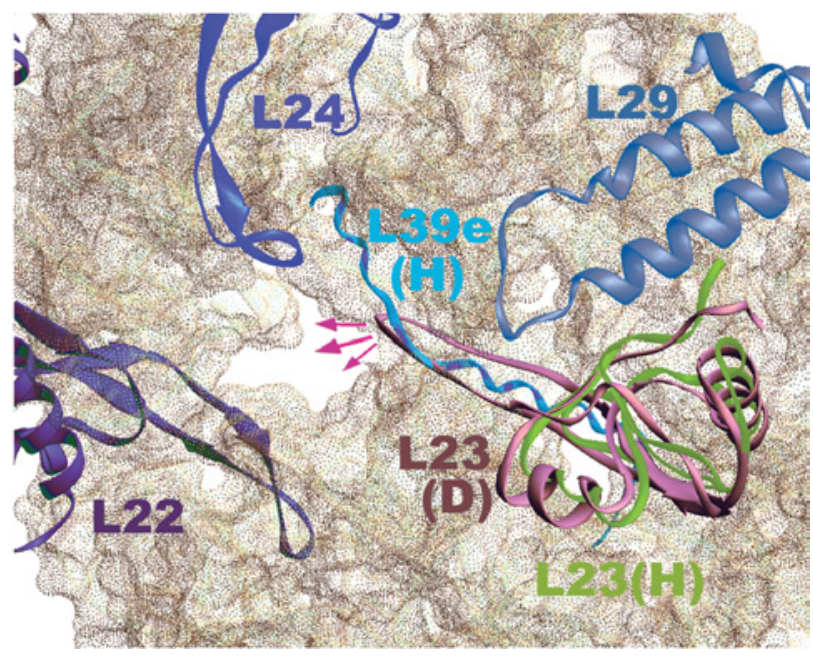

the nucleotide in tRNA position 73, which interacts with the ribosome only through its ribose, displays sequence variability.

\section{Nascent protein progression and co-translational folding}

Recent biochemical studies revealed that, contrary to its previous characteristic as a passive path [8], the exit tunnel functions as a discriminating gate for exporting proteins, responds to cellular signals $[27,28]$ and may control co-translational folding events [22]. Analysis of the crystal structures of complexes of D50S with various compounds bound to the tunnel indicated that most of the tunnel functions can be accomplished by mobile ribosomal proteins, such as L22 and L23, penetrating the tunnel walls, which are composed mainly of ribosomal RNA [5,22,29]. The rotatory motion ensures that nascent proteins tunnel entry at a $\beta$-like conformation, allowing for smooth nascent protein progression. Nevertheless, under specific cellular conditions [30], motions of the nascent protein may become restricted when a proline residue reaches the PTC $[10,29]$ and nascent protein advancement through the tunnel can be hampered by tunnel gating, achieved by protein L22 $\beta$-hairpin tip swing across it $[10,29]$.

The motion of protein L23 extended loop appears to be involved with the trafficking of nascent proteins into their folding space. Protein L23 is placed at the tunnel opening and the TF (trigger factor), the ribosome-associated chaperones in eubacteria, binds to it [31-33]. L23 is among the few r-proteins with significant sequence differences among the three kingdoms of life. Only in eubacteria does it possess a sizable extended loop, which in D50S structure infiltrates the tunnel wall from its exterior into its opening [5]. Crystallographic studies showed almost identical TF binding to the globular domain of protein L23 of D50S [22] and of H50S [34]. However, whereas no conformational changes were observed in H50S by TF binding, significant allosteric conformational alterations at L23 extended the loop accompanying TF binding to D50S (Figure 3). These alterations vary the tunnel shape in a fashion that may control the rate and directionality of nascent protein entry into their folding site. They may also provide a communication path between nascent chains, the TF, the ribosomal tunnel and additional cellular components. The absence of similar allosteric rearrangements in the chimaeric complex built of archaeal H50S with eubacterial TF, is consistent with TF specificity to the eubacteria kingdom. It also indicates that different ribosomal-associated chaperones may bind in a similar way, but their function in assisting protein trafficking into the folding cradle is species- or kingdom-dependent.

We thank the ribosome group at the Weizmann Institute and the former MPG Research Unit in Hamburg. X-ray diffraction data were collected at ID19/SBC/APS/ANL and ID14/ESRF-EMBL. U.S. National Institutes of Health (GM34360), Human Frontier Science Program Organization (HFSP: RGP0076/2003) and Kimmelman Center for Macromolecular Assemblies, provided support.

\section{References}

1 Ban, N., Nissen, P., Hansen, J., Moore, P.B. and Steitz, T.A. (2000) Science 289, 905-920

2 Schluenzen, F., Tocilj, A., Zarivach, R., Harms, J., Gluehmann, M., Janell, D., Bashan, A., Bartels, H., Agmon, I., Franceschi, F. et al. (2000) Cell (Cambridge, Mass.) 102, 615-623

3 Wimberly, B.T., Brodersen, D.E., Clemons, Jr, W.M., Morgan-Warren, R.J., Carter, A.P., Vonrhein, C., Hartsch, T. and Ramakrishnan, V. (2000) Nature (London) 407, 327-339

4 Yusupov, M.M., Yusupova, G.Z., Baucom, A., Lieberman, K., Earnest, T.N., Cate, J.H. and Noller, H.F. (2001) Science 292, 883-896

5 Harms, J., Schluenzen, F., Zarivach, R., Bashan, A., Gat, S., Agmon, I., Bartels, H., Franceschi, F. and Yonath, A. (2001) Cell (Cambridge, Mass.) 107, 679-688

6 Noller, H.F., Hoffarth, V. and Zimniak, L. (1992) Science 256, 1416-1419

7 Nitta, I., Kamada, Y., Noda, H., Ueda, T. and Watanabe, K. (1998) Science 281, 666-669

8 Nissen, P., Hansen, J., Ban, N., Moore, P.B. and Steitz, T.A. (2000) Science 289, 920-930

9 Bashan, A., Agmon, I., Zarivach, R., Schluenzen, F., Harms, J., Berisio, R. Bartels, H., Franceschi, F., Auerbach, T., Hansen, H.A.S. et al. (2003) Mol. Cell 11, 91-102

10 Agmon, I., Auerbach, T., Baram, D., Bartels, H., Bashan, A., Berisio, R., Fucini, P., Hansen, H.A., Harms, J., Kessler, M. et al. (2003) Eur. J. Biochem. 270, 2543-2556

11 Moore, P.B. and Steitz, T.A. (2003) RNA 9, 155-159

12 Polacek, N., Gaynor, M., Yassin, A. and Mankin, A.S. (2001) Nature (London) 411, 498-501

13 Thompson, J., Kim, D.F., O'Connor, M., Lieberman, K.R., Bayfield, M.A Gregory, S.T., Green, R., Noller, H.F. and Dahlberg, A.E. (2001) Proc. Natl. Acad. Sci. U.S.A. 98, 9002-9007

14 Barta, A., Dorner, S. and Polacek, N. (2001) Science 291, 203

15 Bayfield, M.A., Dahlberg, A.E., Schulmeister, U., Dorner, S. and Barta, A. (2001) Proc. Natl. Acad. Sci. U.S.A. 98, 10096-10101

16 Polacek, N., Gomez, M.J., Ito, K., Xiong, L., Nakamura, Y. and Mankin, A. (2003) Mol. Cell 11, 103-112

17 Youngman, E.M., Brunelle, J.L., Kochaniak, A.B. and Green, R. (2004) Cell (Cambridge, Mass.) 117, 589-599 
18 Weinger, J.S., Parnell, K.M., Dorner, S., Green, R. and Strobel, S.A. (2004) Nat. Struct. Mol. Biol. 11, 1101-1106

19 Jencks, W.P. (1969) Catalysis in Chemistry and Enzymology, McGraw-Hill, NY

20 Agmon, I., Bashan, A., Zarivach, R. and Yonath, A. (2005) J. Mol. Biol., in the press

21 Agmon, I., Amit, M., Auerbach, T., Bashan, A., Baram, D., Bartels, H., Berisio, R., Greenberg, I., Harms, J., Hansen, H. et al. (2004) FEBS Lett, 567, 20-26

22 Baram, D. and Yonath, A. (2005) FEBS Lett. 579, 948-954

23 Yonath, A. (2003) Biol. Chem. 384, 1411-1419

24 Carter, P. and Wells, J.A. (1987) Science 237, 394-399

25 Dall'Acqua, W. and Carter, P. (2000) Protein Sci. 9, 1-9

26 Aronson, H.E., Royer, Jr, W.E. and Hendrickson, W.A. (1994) Protein Sci. 3, $1706-1711$
27 Woolhead, C.A., McCormick, P.J. and Johnson, A.E. (2004) Cell (Cambridge, Mass.) 116, 725-736

28 Etchells, S.A. and Hartl, F.U. (2004) Nat. Struct. Mol. Biol. 11, 391-392

29 Berisio, R., Schluenzen, F., Harms, J., Bashan, A., Auerbach, T., Baram, D. and Yonath, A. (2003) Nat. Struct. Biol. 10, 366-370

30 Nakatogawa, H. and Ito, K. (2002) Cell (Cambridge, Mass.) 108, 629-636

31 Frydman, J. (2001) Annu. Rev. Biochem. 70, 603-647

32 Hartl, F.U. and Hayer-Hartl, M. (2002) Science 295, 1852-1858

33 Kramer, G., Rauch, T., Rist, W., Vorderwulbecke, S., Patzelt, H., Schulze-Specking, A., Ban, N., Deuerling, E. and Bukau, B. (2002) Nature (London) 419, 171-174

34 Ferbitz, L., Maier, T., Patzelt, H., Bukau, B., Deuerling, E. and Ban, N. (2004) Nature (London) 431, 590-596

Received 20 January 2005 